\title{
Fascitis necrosante grave por Serratia marcescens: Reporte de un caso clínico
}

\author{
Alejandro Campos G., Ana M. Burgos L., Alberto Fica C., Gonzalo Victoriano R., \\ M. Carolina Sarmiento G. y Sandra Osorio V.
}

\section{Fatal necrotizing fasciitis due to Serratia marcescens}

An 81 year old female patient with chronic heart failure and atrial fibrillation receiving anticoagulant therapy, was admitted with progressive pain on her right leg for the past 24 hours, associated to local erythema, edema and warmth. The lesion evolved at the same site where she presented a chronic ulcer for the previous 5 months managed only with local care. At admission a necrotic plaque on the affected site was perceived; there was no hypotension or mental confusion but signs of a deep venous thrombosis on the involved leg were found. She was febrile $\left(37.8^{\circ} \mathrm{C}\right)$ and with tachychardia (126 per minute). Laboratory evaluation revealed normal white blood cell count and a subtherapheutic anticoagulant INR value. A chest $\mathrm{x}-$ ray showed infiltrates on the left lower lung lobe. On the following hours the lesion evolved with increasing pain, haemorrhagic bullae and a purulent discharge through the ulcer, with the patient developing mental deterioration, hypotension, respiratory failure and shock. The patient received intravenous ciprofloxacin and clindamycin and was operated 15 hours after admission performing an over-the knee amputation. A cardiac catheterization demonstrated a low cardiac output $(2.3 \mathrm{~L} / \mathrm{min})$, and both a high systemic vascular resistance (2888 din.s. $\left.\mathrm{cm}^{-5}\right)$ and pulmonary capillary wedge pressure $\left(17 \mathrm{~cm} \mathrm{H}_{2} \mathrm{O}\right)$, results compatible with cardiogenic shock. Evolution was progressively worse and she died of multiple organic failure 36 hours after admission. Two blood culture samples grew Serratia marcescens. No necropsy was performed and cultures taken from the leg remained negative.

Key words: cellulitis, necrotizing fasciitis, Serratia.

Palabras claves: celulitis, fascitis necrosante, Serratia.

\section{Introducción}

$\mathbf{L}$ a fascitis necrosante es una infección poco frecuente que se caracteriza por necrosis del tejido subcutáneo y la fascia, con trombosis de la microcirculación. Puede progresar rápidamente con toxicidad sistémica y, eventualmente, causar la muerte si no se diagnostica y trata en forma rápida. La diferenciación con infecciones frecuentes como celulitis y abscesos es muy importante. Tradicionalmente, esta infección ha sido ligada a algunas variantes virulentas de Streptococcus pyogenes y, rara vez, a bacilos gramnegativos. Recientemente, observamos un caso fulminante asociado a Serratia marcescens que nos pareció de interés comunicar.

\section{Caso clínico}

Paciente de sexo femenino, de 81 años, con antecedentes de hipertensión arterial, insuficiencia cardíaca
(CF II NYHA), valvulopatía mitro-aórtica, cardiopatía dilatada y fibrilación auricular. La paciente estaba en tratamiento anticoagulante oral, con beta-bloqueadores e inhibidores del receptor de angiotensina II, y durante el año 2006, acudió en el período de cinco meses, a controles y curaciones por una lesión ulcerada crónica en el tercio medio de la pierna derecha. Su última hospitalización había ocurrido en septiembre de 2003 por descompensación de su condición cardíaca.

La paciente ingresó a comienzos de mayo de 2006, por un cuadro fulminante de 24 horas de evolución, con dolor progresivo en la pierna derecha, aumento de volumen y eritema en torno a la lesión ulcerada. Al examen físico destacaba la presencia de fiebre $\left(37,6^{\circ} \mathrm{C}\right.$ axilar), taquicardia (126 latidos por min) y un marcado edema en la pierna derecha, con calor local y una lesión ulcerada necrótica con halo eritematoso de 10 por $5 \mathrm{~cm}$, en el tercio medio de la cara lateral externa. No había hipotensión arterial, compromiso de conciencia, ritmo de galope ni congestión pulmonar al ingreso. Los exámenes iniciales indicaron: leucocitos

\author{
Hospital Clínico \\ Universidad de Chile, Santiago \\ Chile \\ Departamento de Cirugía (ACG, \\ AMBL, GVR, MCSG, SOV) \\ Departamento de Medicina \\ Sección de Infectología (AFC) \\ Recibido: 5 de diciembre 2006 \\ Aceptado: 14 de mayo 2007 \\ Correspondencia a: \\ Alberto Fica Cubillos \\ email:afica@redclinicauchile.cl
}


en recuento de $6.310 / \mathrm{mm}^{3}$, con desviación a izquierda (10\% de baciliformes). Una evaluación con ecografía doppler demostró una trombosis venosa profunda poplítea y del grupo gemelar interno derecho con moderado edema celular subcutáneo. El tiempo de protrombina estaba prolongado $\left(20,9^{\prime \prime}\right)$ con un valor INR de 1,92 (subterapéutico). Una radiografía de tórax demostró imágenes de consolidación en el lóbulo pulmonar inferior izquierdo.

En las horas siguientes a su ingreso, la lesión progresó con mayor dolor y aparición de bulas con contenido hemorrágico y salida de secreción purulenta por la úlcera de la pierna. Desarrolló compromiso de conciencia, hipotensión arterial, falla respiratoria y luego shock.

Se intervino quirúrgicamente a las 15 horas de su ingreso con el diagnóstico de fascitis necrosante, efectuándosele amputación supracondílea de la extremidad comprometida. No se tomaron cultivos de tejido. Se observaba una extremidad violácea, con bulas hemorrágicas y zonas de necrosis y mal olor. En la zona de la amputación había abundante edema en el tejido adiposo y se observó un escaso sangrado en ese sitio. Desde su ingreso, la paciente recibió tratamiento antimicrobiano con ciprofloxacina $400 \mathrm{mg} \mathrm{c} / 12$ horas ev y clindamicina $600 \mathrm{mg} \mathrm{c} / 8$ horas ev.

En el post-operatorio evolucionó en forma inestable, siendo necesario el uso de fármacos vasoactivos y soporte ventilatorio, desarrollando una falla renal aguda con anuria, acidosis metabólica grave y coagulopatía. La cateterización hemodinámica objetivó un shock cardiogénico con gasto cardíaco bajo (2,3 L/min), aumento de la resistencia vascular sistémica $(2.888$ din.s.cm $\left.{ }^{-5}\right)$ y de la presión capilar pulmonar $(17 \mathrm{~cm}$ de agua). La presión arterial pulmonar estaba elevada $(57 / 40 \mathrm{mmHg})$. La paciente falleció a las 36 horas del ingreso por falla orgánica múltiple. Los hemocultivos obtenidos al ingreso demostraron la presencia de Serratia marcescens (sensible a ciprofloxacina, aminoglucósidos, cotrimoxazol, carbapenémicos y resistente a ampicilina y cefalosporinas de primera generación). El cultivo obtenido de la secreción del muslo derecho, antes de la cirugía, fue negativo. No se efectuó necropsia.

\section{Discusión}

Las infecciones de piel y tejidos blandos de las extremidades incluyen una variedad de condiciones de diferente gravedad y pronóstico. Dentro de ellas, los cuadros de fascitis necrosante ocupan un lugar relevante por su evolución fulminante y alta letalidad.

La fascitis necrosante es definida por la necrosis de la fascia y del tejido subcutáneo y se asocia a un daño de la microcirculación por trombosis. La infección genera necrosis tisular y activa la cascada de la coagulación que produce trombosis local e infarto. Ello explica el intenso dolor que sufren estos pacientes y la ausencia de hemorragia encontrada en las intervenciones quirúrgicas. A medida que la infección progresa, la presión local aumenta, comprometiendo más la irrigación vascular y permitiendo el desarrollo de un síndrome compartamental con síntomas vasculares y neurológicos ${ }^{1}$.

La fascitis necrosante puede afectar cualquier parte del cuerpo, pero es más frecuente el compromiso de extremidades, periné y tronco. La mayoría de los pacientes presenta signos locales de inflamación como eritema, induración y dolor del sitio afectado, asociados a fiebre y taquicardia; el dolor puede ser desproporcionado a las manifestaciones locales y acompañado de signos de toxicidad sistémica; discordancia que permite sospechar este cuadro. Sin embargo, al inicio, los síntomas suelen ser inespecíficos y el diagnóstico realizado en forma tardía. A medida que la infección progresa, la piel se hace cada vez más tensa y eritematosa, sin márgenes definidos, se produce un cambio de color secuencial de rojo a púrpura y después azulado, hasta progresar a necrosis con formación de bulas que pueden ser hemorrágicas. Los pacientes con estadios avanzados desarrollan sepsis grave y shock séptico, que puede comenzar con confusión, agitación, o compromiso de conciencia cuantitativo, progresando, rápidamente, a falla multiorgánica. Es frecuente encontrar en los análisis de laboratorio leucocitosis, hiperglicemia, alteración de la función renal, hipoalbuminemia, acidosis y alteración de las pruebas de coagulación.

La TAC y la RM son usadas cuando el diagnóstico es dudoso; ambos tienen un rol en delimitar la extensión de la infección y mostrar la presencia de gas en los tejidos blandos, pero la condición crítica de estos pacientes, con frecuencia impide su realización y sólo retrasa el tratamiento. Por lo tanto, el diagnóstico sigue siendo principalmente clínico. Lamentablemente, en ocasiones, la confirmación de la fascitis necrosante se realiza sólo al momento de la exploración quirúrgica y en forma tardía.

Debido a que los elementos clínicos suelen ser inespecíficos, especialmente en las horas iniciales, la sospecha a tiempo constituye el eje para lograr un tratamiento oportuno. Varios elementos clínicos han sido sugeridos para una sospecha o reconocimiento precoz (Tabla 1).

Desde el punto de vista etiológico, la mayor parte de los cuadros de fascitis necrosante $(\sim 50 \%)$ son infecciones polimicrobianas que involucran bacilos 
gramnegativos, anaerobios y Streptococcus no pyogenes ${ }^{1-3}$. Por su parte, las fascitis necrosantes monomicrobianas están asociadas, predominantemente, a $S$. pyogenes; las no asociadas a $S$. pyogenes son muy infrecuentes y han sido relacionadas a Streptococcus agalactiae, Klebsiella sp. y Vibrio vulnificus ${ }^{1,2}$.

Hasta ahora, pocos casos de celulitis o fascitis necrosante por $S$. marcescens han sido descritos en la literatura, reflejando lo infrecuente de esta condición. Los casos reportados corresponden mayoritariamente a celulitis (aproximadamente el $80 \%$ ) y el resto a fascitis. En su mayoría, se trata de pacientes con alguna condición crónica, tal como diabetes mellitus, cardiopatías, insuficiencia renal o inmunosupresión; o la existencia de un traumatismo previo, mordedura por mascotas o úlceras de extremidades ${ }^{4-7}$. Algunos casos no se presentan con una puerta de entrada identificable. El rango etario es amplio, incluyendo niños y ancianos ${ }^{4,8}$.

Las infecciones reportadas para $S$. marcescens incluyen cuadros fulminantes, gran compromiso de piel $\mathrm{y}$ tejidos blandos, aparición de bulas hemorrágicas $\mathrm{y}$, en los casos más graves, fascitis y shock $k^{4,8,9}$. Las extremidades están afectadas en casi todos los casos y, excepcionalmente, otros sitios como el cuello ${ }^{8}$. La participación de $S$. marcescens como agente causal ha sido demostrada por su presencia en hemocultivos o en cultivos de las lesiones, ocasionalmente con cultivos polimicrobianos ${ }^{4,6,8,10}$. La ausencia de bacterias anaerobias en estos cultivos es universal.

Las infecciones por $S$. marcescens son más conocidas por su perfil hospitalario, asociadas a eventos nosocomiales en pacientes vulnerables, en forma esporádica o en brotes y no por su génesis en la comunidad, como sucedió en este caso. Por ejemplo, en un brote descrito en nuestro país, las infecciones estuvieron ligadas a infección urinaria, neumonía, infección de herida operatoria, bacteriemia $u$ otras condiciones en pacientes hospitalizados con neoplasias, diabetes mellitus, alcoholismo, insuficiencia cardiaca o renal. ${ }^{11}$ En este caso, se pudo descartar el origen nosocomial de la infección por la ausencia de hospitalizaciones recientes y el perfil, en general susceptible, del microorganismo.

El manejo de estos cuadros está basado en la sospecha temprana, la exploración quirúrgica precoz, aseos frecuentes, antimicrobianos parenterales de amplio espectro, soporte intensivo y, en el caso de infecciones invasoras por $S$. pyogenes, el uso de inmunoglobulina humana endovenosa para contrarrestar el efecto de los superantígenos ${ }^{12}$. Es muy importante que se integren adecuadamente todos estos elementos en forma oportuna. En la sospecha precoz se puede mencionar una estrategia muy útil que puede ser desarrollada al lado del paciente. Mediante una incisión con

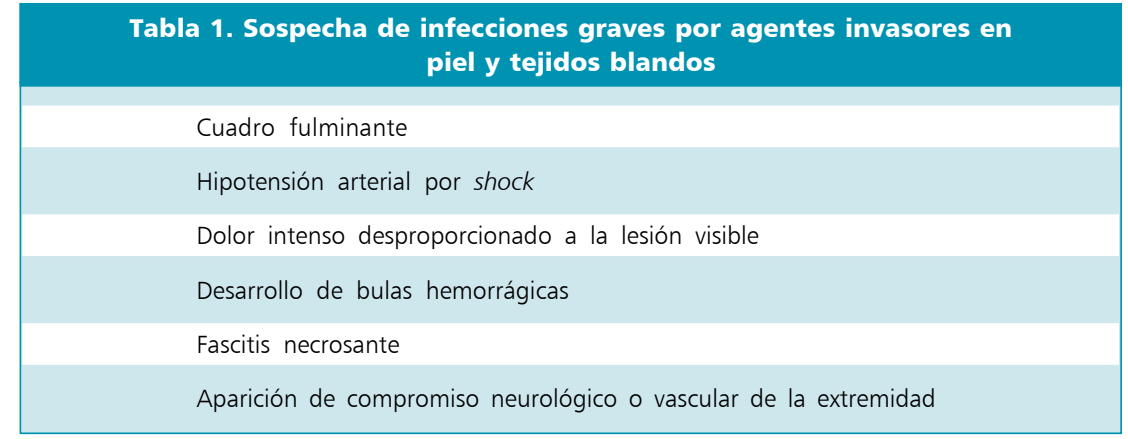

bisturí (previa anestesia local), apreciar si se puede separar digitalmente el tejido adiposo de la aponeurosis en forma fácil. En los casos de fascitis necrosante, no hay resistencia tisular a esta maniobra, pudiéndose apreciar, además, un tejido que sangra muy poco.

El pronóstico de estas infecciones es favorable en aproximadamente $80 \%$ de los casos, pero de alta letalidad en casos de fascitis $(50 \% \text { o más })^{4,9}$. El tratamiento antimicrobiano con compuestos activos, los aseos quirúrgicos amplios o, incluso, la amputación de la extremidad afectada, no han podido revertir el mal pronóstico de las formas con fascitis por Serratia ${ }^{4,9}$.

Se debe destacar que no todos los cuadros de shock presentes en una infección son necesariamente de tipo distributivo. Por ejemplo, en nuestra paciente, la insuficiencia cardíaca pre-existente y la probable embolia pulmonar asociada, determinaron la presencia de un shock cardiogénico. La sospecha de embolia pulmonar queda sugerida por la asociación de trombosis venosa profunda con imágenes pulmonares y un tratamiento anticoagulante sub-terapéutico. La distinción del tipo de shock es importante por el tipo de terapia de soporte y la potencial reversibilidad de la condición de base. Aunque la infección no fue la causa directa de la muerte en nuestra paciente, ella permitió una descompensación irreversible en una paciente con una pobre reserva cardiovascular de base.

El caso reportado ilustra el diagnóstico diferencial que existe en los cuadros de fascitis necrosante, la importancia de la toma de hemocultivos, la gravedad intrínseca de esta condición y, aunque en este caso lamentablemente sin resultados favorables, la necesidad básica de un enfoque multidisciplinario.

\section{Resumen}

Una paciente de 81 años con insuficiencia cardíaca crónica, fibrilación auricular y tratamiento anticoagulante, ingresó por un cuadro fulminante de dolor y 
celulitis en la extremidad inferior derecha de 24 horas de evolución. Sobre la zona existía una úlcera crónica de cinco meses de evolución, manejada con curaciones locales. Al ingreso, había una placa necrótica pero sin hipotensión o confusión mental. La paciente estaba febril y con taquicardia (126 por min). La evaluación reveló ausencia de leucocitosis, trombosis venosa profunda en la misma pierna e infiltrados radiológicos pulmonares en el lóbulo inferior izquierdo. En las horas siguientes aumentó el dolor, apareció secreción purulenta por la úlcera y la paciente presentó confusión, hipotensión, falla respiratoria y luego shock.
La paciente recibió ciprofloxacino endovenoso y clindamicina y fue intervenida a las 15 horas de ingreso, efectuándose una amputación supracondílea. El sondeo cardíaco demostró un gasto bajo $(2,3 \mathrm{~L} / \mathrm{min})$ y una resistencia vascular sistémica $\left(2888\right.$ din.s.cm $\left.{ }^{-5}\right)$ y presión capilar pulmonar elevada $\left(17 \mathrm{~cm} \mathrm{H}_{2} \mathrm{O}\right)$, cifras compatibles con un shock cardiogénico. Evolucionó en malas condiciones y falleció de falla orgánica múltiple a las 36 horas de ingreso. Los hemocultivos demostraron crecimiento de Serratia marcescens en dos frascos. No se efectuó una necropsia y los cultivos de la secreción de la úlcera fueron negativos.

\section{Referencias}

1.- Urschel J D. Necrotizing soft tissue infections. Postgrad Med J 1999; 75: 645-9.

2.- Wong C H, Chang H C, Pasupathy S, Khin L W, Tan J L, Low C O. Necrotizing fasciitis: Clinical presentation, microbiology, and determinants of mortality; J Bone Joint Surg Am 2003; 85-A: 1454-60.

3.- Hasham S, Matteucci P, Stanley P R W, Hart N B. Necrotising fasciitis. BMJ 2005; 330: $830-3$.

4.- Liangpunsakul S, Pursell K. Communityacquired necrotizing fasciitis caused by Serratia marcescens: Case report and review. Eur J Clin Microbiol Infect Dis 2001; 20:
509-21.

5.- Bachmeyer C, Sanguina M, Turc Y, Reynaert G, Blum L. Necrotizing fasciitis due to Serratia marcescens. Clin Exp Dermatol 2004; 29: 673-4.

6.- Hiesh S, Babl F E. Serratia marcescens cellulitis following an Iguana bite. Clin Infect Dis 1999; 28: 1181-2.

7.- Bonner M J, Meharg Jr J G. Primary cellulitis due to Serratia marcescens. JAMA 1983; 250: 2348-9.

8.- Newton C L, deLemos D, Abramo T J, Murrey A, Noell C. Cervical necrotizing fasciitis caused by Serratia marcescens in a 2 years old. Pediatr Emerg 2002; 18: 433-5.

9.- Curtis C E, Chock S, Henderson T, Holman M J. A fatal case of necrotizing fasciitis caused by Serratia marcescens. Am Surg 2005; 71: 228-30.

10.- Cooper C L, Wiseman M, Brunham R. Bullous cellulitis caused by Serratia marcescens. Int J Infect Dis 1998; 3: 36-8.

11.- Dossi M T, Escalona M, Serrano C, Silva M A, Juliet C, Fernández A, et al. Serratia marcescens. Descripción de un brote de infección intrahospitalaria. Rev Chil Infectol 2002; 19: 262-6.

12.- Kaul R, McGeer A, Norrby-Teglund A, Kotb M, Schwartz B, O'Rourke K, et al. Intravenous immunoglobulin therapy for streptococcal toxic shock syndrome. A comparative observational study. Clin Infect Dis 1999; 28: 800-7. 